Ann. Geophysicae 18, 28-41 (2000) (C) EGS - Springer-Verlag 2000

\title{
Energy time dispersion of a new class of magnetospheric ion events observed near the Earth's bow shock
}

\author{
G. C. Anagnostopoulos ${ }^{1}$, N. Paschalidis ${ }^{1,2}$, A. N. Littas $^{1}$ \\ ${ }^{1}$ Department of Electrical and Computer Engineering, University of Thrace, Xanthi 67100, Greece \\ ${ }^{2}$ Applied Physics Laboratory, The Johns Hopkins University, Laurel, MD 20723-6099, USA
}

Received: 18 November 1998 / Revised: 7 June 1999 / Accepted: 9 June 1999

\begin{abstract}
We have analyzed high time resolution ( $\geq 6 \mathrm{~s})$ data during the onset and the decay phase of several energetic $(\geq 35 \mathrm{keV})$ ion events observed near the Earth's bow shock by the CCE/AMPTE and IMP-7/8 spacecraft, during times of intense substorm/geomagnetic activity. We found that forward energy dispersion at the onset of events (earlier increase of middle energy ions) and/or a delayed fall of the middle energy ion fluxes at the end of events are often evident in high time resolution data. The energy spectra at the onset and the decay of this kind of events show a characteristic hump at middle (50-120 keV) energies and the angular distributions display either anisotropic or broad forms. The time scale of energy dispersion in the ion events examined was found to range from several seconds to $\sim 1 \mathrm{~h}$ depending on the ion energies compared and on the rate of variation of the Interplanetary Magnetic Field (IMF) direction. Several canditate processes are discussed to explain the observations and it is suggested that a rigidity dependent transport process of magnetospheric particles within the magnetosheath is most probably responsible for the detection of this new type of near bow shock magnetospheric ion events. The new class of ion events was observed within both the magnetosheath and the upstream region.
\end{abstract}

Key words. Interplanetary physics (energetic particles; planetary bow shocks)

\section{Introduction}

The terrestrial magnetosphere has been known for many years to be an important source of energetic $(\geq 30 \mathrm{keV})$

Correspondence to: G. Anagnostopoulos

E-mail: anagnosto@xanthi.cc.duth.gr ions in the magnetosheath (Williams et al., 1988; Kudela et al., 1992; Paschalidis et al., 1994; Karanikola et al., 1999 ) and the near Earth interplanetary space (Sarris et al., 1978; Anagnostopoulos et al., 1986; 1999). It is also known that different estimations for the extent of contribution of the magnetospheric ion population in the magnetosheath and the upstream region have been postulated (Scholer et al., 1981; Anagnostopoulos et al., 1986). It has been suggested that acceleration of solar wind ions at or near the bow shock, is another principal source, beside the magnetospheric one, that can explain the presence of energetic ions.

Shock drift acceleration (SDA) successfully explains some of the field aligned beams (FABs) of ions in the upstream region and some of the ion events displaying double peaked anisotropies in the magnetosheath at energies from some $\mathrm{keV}$ up to a few $\mathrm{MeV}$ (Giacalone, 1992; Anagnostopoulos and Kaliabetsos, 1994). These ion events are observed near the quasi-perpendicular side of the bow shock.

Diffusive (Fermi) acceleration was proposed as an explanation for an ion population with broad angular distribution often observed at positions magnetically connected with the quasi-parallel (dawn) side of the bow shock (Lee, 1982; Ellison et al., 1990; Scholer et al., 1992); this population is often called "diffuse" (Gosling et al., 1978). However, since energetic ions of magnetospheric origin after their leakage in the upstream region can scatter at magnetic waves and produce "diffuse" type distributions as well (Sibeck et al., 1988), we will refer to this population by the more general and neutral term "broad angular distribution" (BAD).

The new point of the present study comes from the analysis of high time ( $\sim 6 \mathrm{~s})$ resolution data during the onset and the decay phase of energetic ion events. We found evidence that the BAD ion events, when observed during times of intense substorm/geomagnetic activity, often show at the onset phase earlier increase in the high energy ion intensities than in the lower energy ion intensities (forward energy dispersion). Furthermore, longer flux-time profiles of higher energy ions were 
detected not only at the onset, but also during the decay phase of the ion events. The energy dispersion of the ion intensities at the onset and at the end of the near bow shock ion events is related to a special kind of energy spectrum showing a peak at medium energies.

\section{Observations}

We present observations which are representative of a special kind of magnetospheric ion events in the vicinity of the Earth's bow shock. The first series of events we will discuss (A: November 1, 1984) concerns a compressed magnetosphere and it has already attracted the interest of a great number of authors (Baker et al., 1988; Sibeck et al., 1988; Ipavich et al., 1988; Takahashi et al., 1988); we further elaborate this period in order to emphasize the importance of using high time resolution $(6 \mathrm{~s})$ to discover this new kind of magnetospheric ion events near the bow shock.

The second period (B: November 9, 1975) was chosen to contain information which improves the possibility of generalizing the conclusions from the analysis of the ion events on November 1, 1984. For instance, the November 1975 upstream event: (a) extends the time scale of the forward velocity dispersion up to $\sim 1 \mathrm{~h}$ (instead of seconds in case A; (b) demonstrates that the new picture of the upstream ion events is valid in the magnetosheath as well, and (c) shows that north-south IMF turnings can produce similar upstream and downstream ion characteristics as for the dawn-dusk turnings.

The observations presented in this section are from the charged particle instruments and the magnetometers on board the spacecraft CCE/AMPTE and IMP-8. Since the description of these instruments has been presented in a series of our previous papers (Anagnostopoulos et al., 1986; Paschalidis et al., 1994, and references therein), we will not repeat it here.

\subsection{The November 1, 1984 ion events}

Figure 1 shows high time (6 s) resolution magnetic field and ion data from the CCE spacecraft for the time interval 0648-0700 UT on November 1, 1984. During that interval CCE observed two upstream events: the event marked a and the onset of the event marked $b$. The inset in Fig. 1 displays the projections of the spacecraft CCE/AMPTE, IMP-8 and of the geostationary spacecraft 1984-037 on the ecliptic plane at $\sim 1720$ UT, on November 1, 1984. Also displayed in the same figure are the assumed positions of the bow shock and of the magnetopause at that time.

Figure 1 shows that the CCE spacecraft left the magnetosheath at 0649:50 UT and that after that time it detected small increases of low energy $(35-71 \mathrm{keV})$ ion intensities. A variation of the IMF direction from $\varphi \cong 90^{\circ}$ toward the radial direction at later times was accompanied by the appearance of strong upstream ion and wave activity. The onset of the 49-71 keV and 115$215 \mathrm{keV}$ ion fluxes was detected by CCE at $\sim 0651 \mathrm{UT}$, whereas the onset of the $35-45 \mathrm{keV}$ ions was detected $\sim 40$ s later, at $\sim 0651: 40 \mathrm{UT}$, displaying a clear forward energy (velocity) dispersion (abbreviated: FED/FVD). The 49-71 keV and the 115-215 keV ions show a peakto-background flux ratio $\mathrm{p} / \mathrm{b} \geq 100$ and $\mathrm{p} / \mathrm{b} \geq 6$ respectively, at the onset. These onsets were detected upstream from a quasi-perpendicular region of the bow shock as implied from the stable profile of the shock and the direction of the magnetic field (almost perpendicular to the Sun-Earth line). The 35-49 keV ions appeared at the time of a major variation of the IMF longitude $(\sim 0652$ UT) and reached a peak-to-background ratio $\mathrm{p} / \mathrm{b}$ as high as $\sim 18$ within a short time period of $\sim 20 \mathrm{~s}$. Although a better time resolution of the data would have given more reliable results, it looks as though the 35-49 keV ion enhancement precedes the appearance of the upstream magnetic field turbulence, which was observed after $\sim 0652$ UT; we note that such behavior was also confirmed for the other two upstream events marked $b$ in Fig. 1 and $c$ in Fig. 3.

As long as the IMF longitude was changing, the flux level of the low energy $(35-49 \mathrm{keV})$ ions after the appearance of the waves increased only slightly, i.e. a factor of $\sim 2$ with respect to the ion flux at 0652 UT. Therefore, we conclude that the wave activity affected the flux level of the low energy ions a little or, perhaps, not at all. Such a small flux increase associated with the appearance of waves suggests that the low energy ions after 06:52 UT most probably have the same source as the ions observed at 06:52 UT. As a consequence of the generation of the wave activity, the angular distributions altered and showed a BAD type ion event at that time (Sibeck et al., 1988; their Fig. 6).

The middle energy ions reached high flux levels with a $\mathrm{p} / \mathrm{b}$ ratio as high as $\geq 100$ (1000) before (after) 06:52 UT. In contrast to the flat top of the low energy ions, the higher $(\geq 115 \mathrm{keV})$ energy ion fluxes increased monotonically from the beginning of the event to $\sim 0652: 30$ UT. (Here we should note that if only the 35-49 keV and the high 215-407 keV ion channels were compared, they would give the impression of inverse velocity dispersion).

The appearance of the middle energy (49-215 keV) ions before the appearance of the lower energy ions (35$49 \mathrm{keV}$ ) and of the wave activity, and the detection of lower $\mathrm{p} / \mathrm{b}$ values in the lowest energy channel (35$49 \mathrm{keV}$ ) suggest that Fermi acceleration is not a source of the higher energy ions (49-215 keV) up to 0652 UT. Furthermore, the presence of higher energy ions cannot be attributed to shock drift acceleration (SDA) for the following reasons: (a) SDA can accelerate ions up to energies $\mathrm{E} \geq 10 \mathrm{keV}$ only in the presence of a solar ambient energetic particle population (Anagnostopoulos and Kaliabetsos, 1994; Anagnostopoulos, 1994), which does not seem to be the case for the time period considered; (b) SDA cannot explain ion enhancements with ratio $\mathrm{p} / \mathrm{b}$ as high as $\geq 100$ (1000) like the ones observed by CCE before (after) 06:52 UT (see Discussion); and (c) SDA produces at best $<10$ peak-tobackground flux ratio at energies $\mathrm{E} \geq 50 \mathrm{keV}$ (Anagnostopoulos and Kaliabetsos, 1994). 


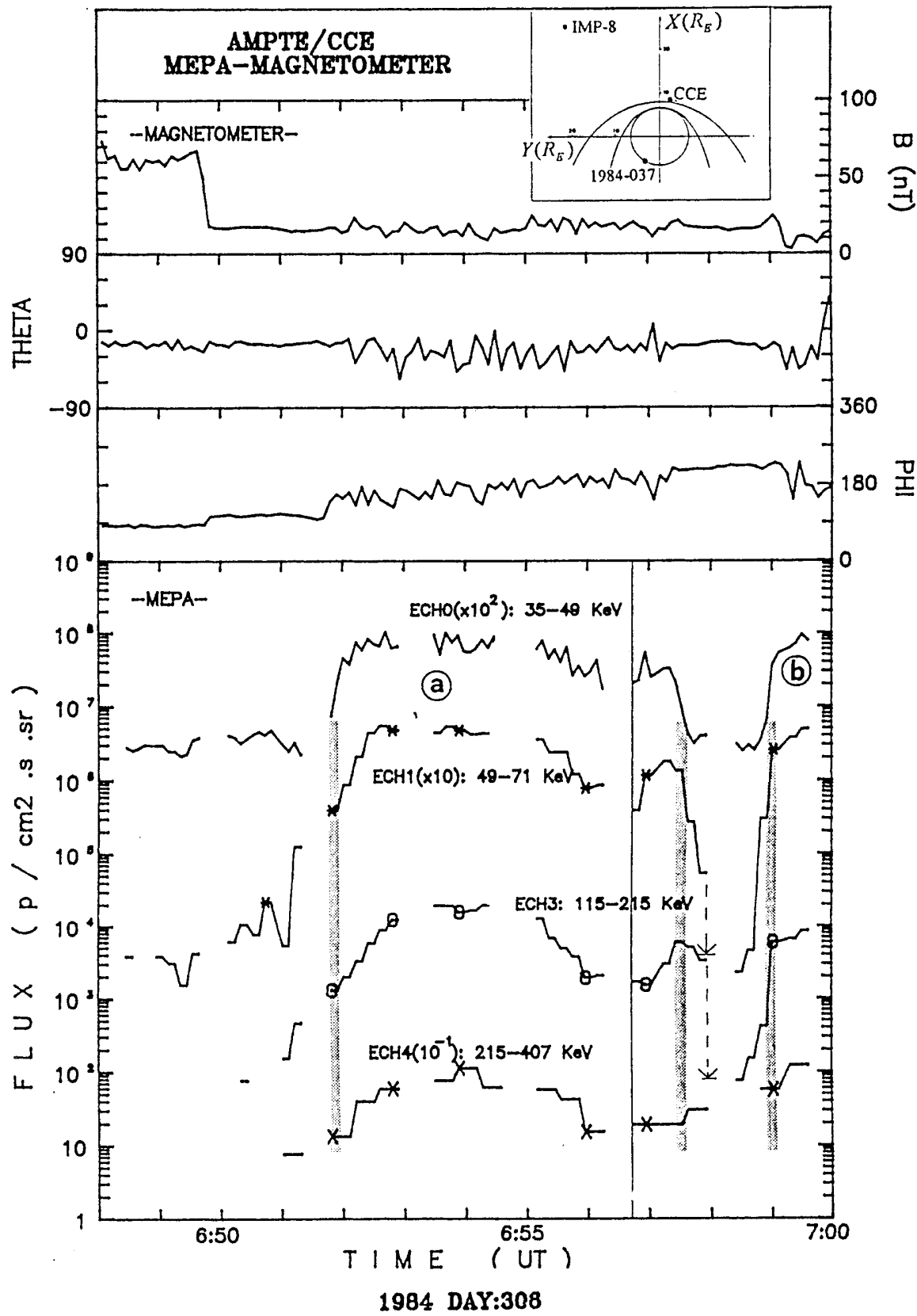

Fig. 1. High time resolution $(\sim 6 \mathrm{~s}) \mathrm{mag}$ netic field and energetic ion data for the upstream event a as detected by CCE. The projection of positions of the spacecraft CCE/AMPTE, IMP-8 and 1984-037 on the plane $\mathrm{X}_{\mathrm{SE}}=0$ on November 1,1984 ( $1715 \mathrm{UT})$, is shown in the inset. The ion fluxes in the middle energy channels (49$215 \mathrm{keV}$ ) rise in general earlier than the lowest energy channel (35-49 keV) displaying forward velocity dispersion at the onset
The information about the high $(\geq 115 \mathrm{keV})$ energy ions at the beginning of the event (FVD, high peak-tobackground ratios, absence of magnetic turbulence) are consistent with the leakage of magnetospheric ions (Anagnostopoulos et al., 1986, 1999). Furthermore, the stable flux-time profile of the low energy ions and the variable profile of the high energy ions during the main phase of the event (a) are consistent with similar behavior of energetic ions within the magnetosphere (Krimigis et al., 1995). We conclude that the whole behavior of energetic ions observed by $\mathrm{CCE}$ to the middle of the event can be well explained in terms of the leakage model for magnetospheric ions (Sibeck et al., 1988; Baker et al., 1988).

The distinct burst-like profile toward the end of the upstream event (a), i.e., after $~ 0656: 45$ UT, is not related to a variation of the direction of the IMF and it extends after the disappearance of the wave activity; Fermi acceleration, therefore, cannot account for the appearance of ions at that time. On the contrary, this ion burst is well correlated in time with the onset of a substorm injection event detected by the geostationary spacecraft 1984-037 (Baker et al., 1988; their Fig. 3). Since a substorm injection event controls the upstream flux behavior at energies $\mathrm{E} \geq 35 \mathrm{keV}$, it is implied that the magnetosphere was also the source of the low energy $(\geq 35 \mathrm{keV}$ ) upstream ions during that time period. A few seconds after the appearance of the ion burst, at 0657:20 UT, the IMF rotated significantly from $\varphi \cong 180$ toward larger longitudes $(\varphi \cong 220)$ and the field lines passing by CCE moved much farther from the dawn magnetopause (towards the bow shock). As a result of the magnetic 
field reconfiguration, a depression in the ion flux was detected by the spacecraft. This depression was observed at lower energies first, while the middle (115-215 keV) and the high (215-407 keV) energy ion flux continued to increase.

The appearance of energetic ions in the absence of upstream waves and the longer duration of the highenergy ion profile at the end of the event is not consistent with Fermi acceleration. The large ratio $\mathrm{p} / \mathrm{b}$ $(\geq 400)$ of the $\geq 49 \mathrm{keV}$ ions is not consistent with previous observations (Anagnostopoulos and Kaliabetsos, 1994) and theoretical predictions of SDA (see Discussion). However, all of these characteristics are consistent with a magnetospheric source for the upstream ions (Anagnostopoulos and Kaliabetsos, 1994). Furthermore, the magnetospheric origin of the upstream ions is greatly supported by the good correlation of the upstream and the magnetospheric ion observations (i.e. the correlation of a distinct upstream burst with a substorm injection event in the nightside magnetosphere at $\sim 0656: 45$ UT). Finally, a comparison of the magnetic field and of the energetic ion observations suggests that the upstream magnetic field wave activity ceases simultaneously with the fall of the low energy fluxes at the pre-event level and presumably suggests that leaking magnetospheric/magnetosheath ions of low energies produced the wave activity.

Figure 1 shows that a variation of the IMF longitude at 0659 UT was followed by the appearance of the upstream event (b) and of wave activity. At the position of CCE the ions of higher energies arrived first and the ions of lower energies some seconds later, displaying again FVD. The time delay between the onset of the 35$49 \mathrm{keV}$ and $49-71 \mathrm{keV}$ ions is $\leq 20 \mathrm{~s}$. FVD in the absence of upstream waves cannot be explained in terms of Fermi acceleration nor in terms of shock drift acceleration, because of the high peak-to-background ratio $\mathrm{R} \geq \sim 140$ of the ion fluxes.

In Fig. 2a we display ion spectra in the energy range $\sim 30-300 \mathrm{keV}$ during three successive time periods: (1) a pre-event interval (0648:33-0649:32 UT), (2) the onset phase of the middle (49-115 keV) energy ions (0651:010651:19 UT), and (3) the onset phase of the low energy (35-49 keV) ions (0651:49-0652:02 UT). A hump at middle energies is evident from this figure during the second period (2). During period (3) the energy spectrum takes a power law shape $\left(j \sim \mathrm{E}^{-\gamma}\right)$, with a spectral index $\gamma=5$.1. In Fig. $2 \mathrm{~b}$ spectra for the plateau phase of the low energy ion event and the bottom panel displays spectra for the decay phase are displayed. We see that a hump at middle energies of the spectrum is evident at the end of the event as in the case of the onset phase. We also see that a variety of spectral shapes were detected throughout the event and that some ion spectra resemble the spectra seen by the ISEE spacecraft and reported by Ipavich et al. (1981) and Anderson (1981).

Figures 3, 4 and 5 show observations for the upstream event (c). Figure 3 was constructed in the same way as Fig. 1. From Fig. 3 it is evident that the energetic ions display FVD at the onset of event (c), with a delay of $\sim 30-40$ s between the onset of the 35-49 keV and the 49-71 keV ions. The upstream ion activity at the onset precedes the wave activity, as in the cases of the upstream events (a) and (b), and is consistent with a magnetospheric source for the energetic ions. After the onset of the event, the energetic $(\geq 35 \mathrm{keV})$ ion fluxes remained nearly stable for $\sim 4 \mathrm{~min}$, and then, at $\sim 0719$, increased.

Pitch angle distributions of the ion intensities after the relative flux increase at 0719 are shown in Fig. 4 as a function of energy; the solid rhombus in this figure correspond to ions with pitch angles $120^{\circ} \leq \theta \leq 180^{\circ}$, streaming in the Sunward direction, and the open circles correspond to the ions with pitch angles $0^{\circ} \leq \theta \leq 60^{\circ}$, streaming in the Earthward direction. During the time interval examined in Fig. 4 (0720-0724 UT) the IMF was radial and turbulent and the CCE spacecraft was found near an almost parallel bow shock front. The observations of Fig. 4 suggest that there exist two different ion populations upstream from the quasiparallel bow shock: (1) a BAD ion population at energies $E \geq \sim 10 \mathrm{keV}$ and (2) an ion population forming a $\mathrm{FAB}$ at energies $\mathrm{E} \leq \sim 10 \mathrm{keV}$; the flux ratio of the sunward and of the earthward streaming ions are found to be $\rho=\sim 1$ at energies $\sim 10 \leq \mathrm{E} \leq 100 \mathrm{keV}$ and $\rho \leq \sim 100$ at energies $\mathrm{E} \leq \sim 10 \mathrm{keV}$.

Figure 5a shows the energy spectrum of a population escaping between 0710-0713 UT upstream from a quasiperpendicular region of the bow shock, and Fig. $5 \mathrm{~b}$ the spectrum of an ion population observed between 07160719 UT when the spacecraft connected with a variety of bow shock structures (from almost perpendicular to almost parallel) due to a varying IMF direction (Fig. 1); in Fig. $5 b$ the spectrum of Fig. 5a (open circles) is also displayed. By comparing the spectra of Fig. 5b we see that a peak at $\sim 6 \mathrm{keV}$ is a common feature of both spectra. From these spectra we conclude that a lowenergy ion population was escaping at those times, upstream from the bow shock, under a variety of IMF directions and bow shock structures. Since the $\mathrm{E} \leq \sim 6 \mathrm{keV}$ ion population was highly anisotropic along the IMF (data not shown in the figure) and not dependent on the IMF direction/bow shock structure, we infer that this is more consistent with leakage of magnetosheath superthermal ions from an ion source downstream of the bow shock whose density is independent of the angle $\theta_{\mathrm{B}_{\mathrm{n}}}$ (Tanaka et al., 1983; Williams et al., 1988), than with bow shock acceleration (Ipavich et al., 1988). We point out that field aligned beams of higher energy $(\geq 50 \mathrm{keV})$ ions were observed at the onset of all the three November 1, 1984, upstream events of Figs. 1 and $3(\sim 2 \leq \rho \leq \sim 8)$ and at times of intensity enhancements throughout the events (for instance $\sim 8 \leq \rho \leq \sim 15$ at $\sim 0652$ UT and $\sim 40 \leq \rho \leq \sim 100$ at $\sim 0715$ UT) as implied from Fig. 6 of Sibeck et al. (1988).

Figure $5 \mathrm{c}$ shows the ion spectra detected between 0720-0724 UT, when the IMF was radial and the spacecraft connected with a quasi-parallel region of the bow shock, and the spectrum shown in Fig. 5a. By comparing the 0720-0724 UT spectrum with the 0716- 

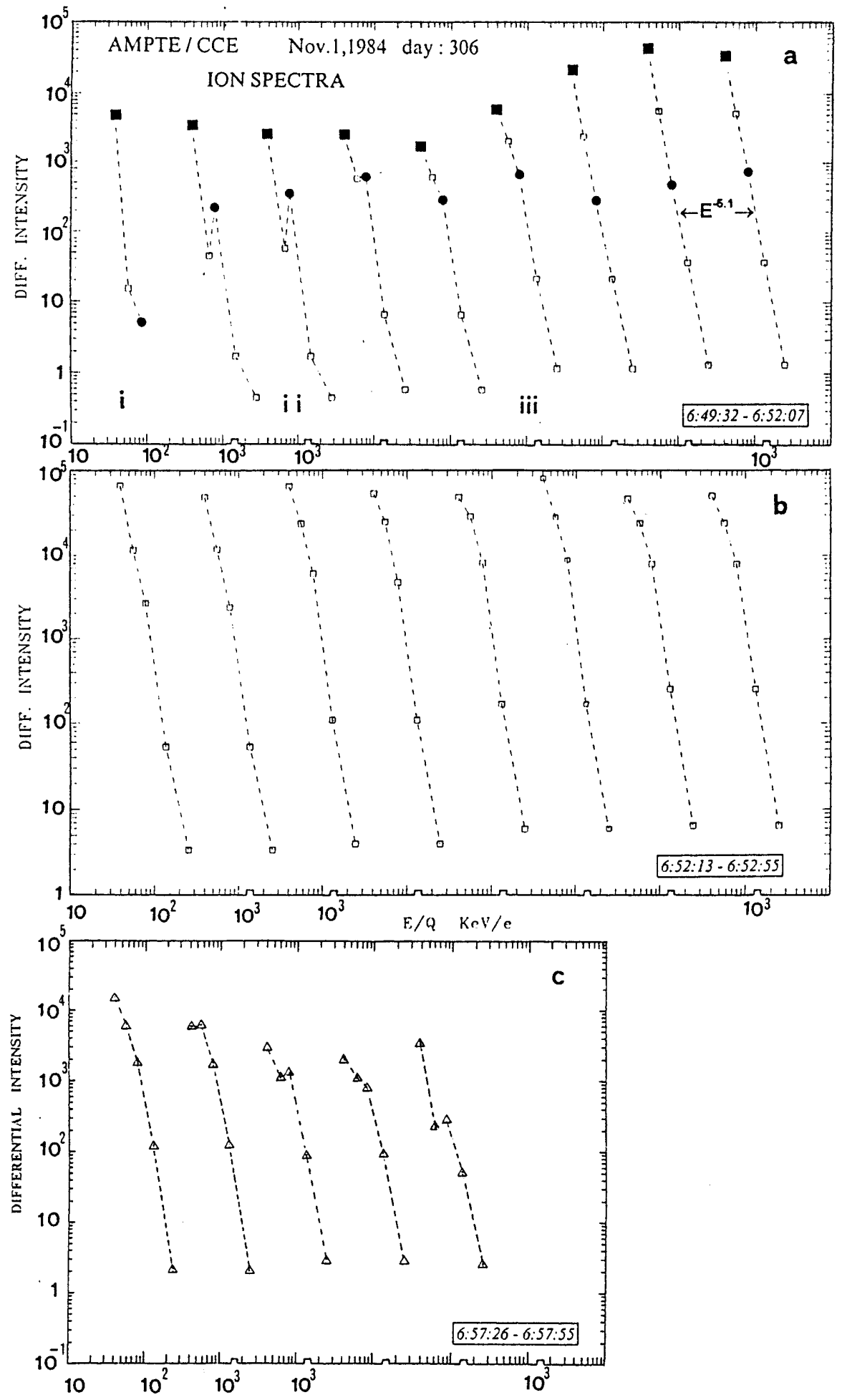

0719 UT can be inferred a remarkable change of the spectral shape of the $\geq \sim 10 \mathrm{keV}$ ion population after $\sim 0720$ UT

Mobius (1990) noted that the upstream 0717-0725 UT ion spectrum is softer than the magnetospheric 0700-0710 UT spectrum at energies $\mathrm{E} \leq 200 \mathrm{keV}$ and he doubted the argument of Baker et al. (1988) and Sibeck et al. (1988) in favor of a magnetopsheric origin of upstream ions at those times. However, there are several
Fig. 2a-c. Successive ion spectra during the onset $\mathbf{a}$, the main $\mathbf{b}$ and the decay phase c of the upstream event (a). A variety of spectral shapes are seen ranging between one with a distinct hump at middle energies (at the very onset and decay phase) and another displaying a power law form in the whole energy range from $\sim 30$ to $\sim 300 \mathrm{keV}$. The series of the spectra reflect different stages in the leakage process of magnetospheric ions due to the IMF variation processes which influence the shape of the spectrum of the leaking ions and that can produce a softer spectrum in the upstream region than the magnetospheric one; such processes are i.e., the energy-dependent drifting of ions moving within the magnetosphere (Takahashi and Iyemori, 1989; Paschalidis et al., 1994), a rigidity dependent propagation of ions within the magnetosheath (Anagnostopoulos et al., 1995, 1999) and the influence of the bow shock on the escaping ions 


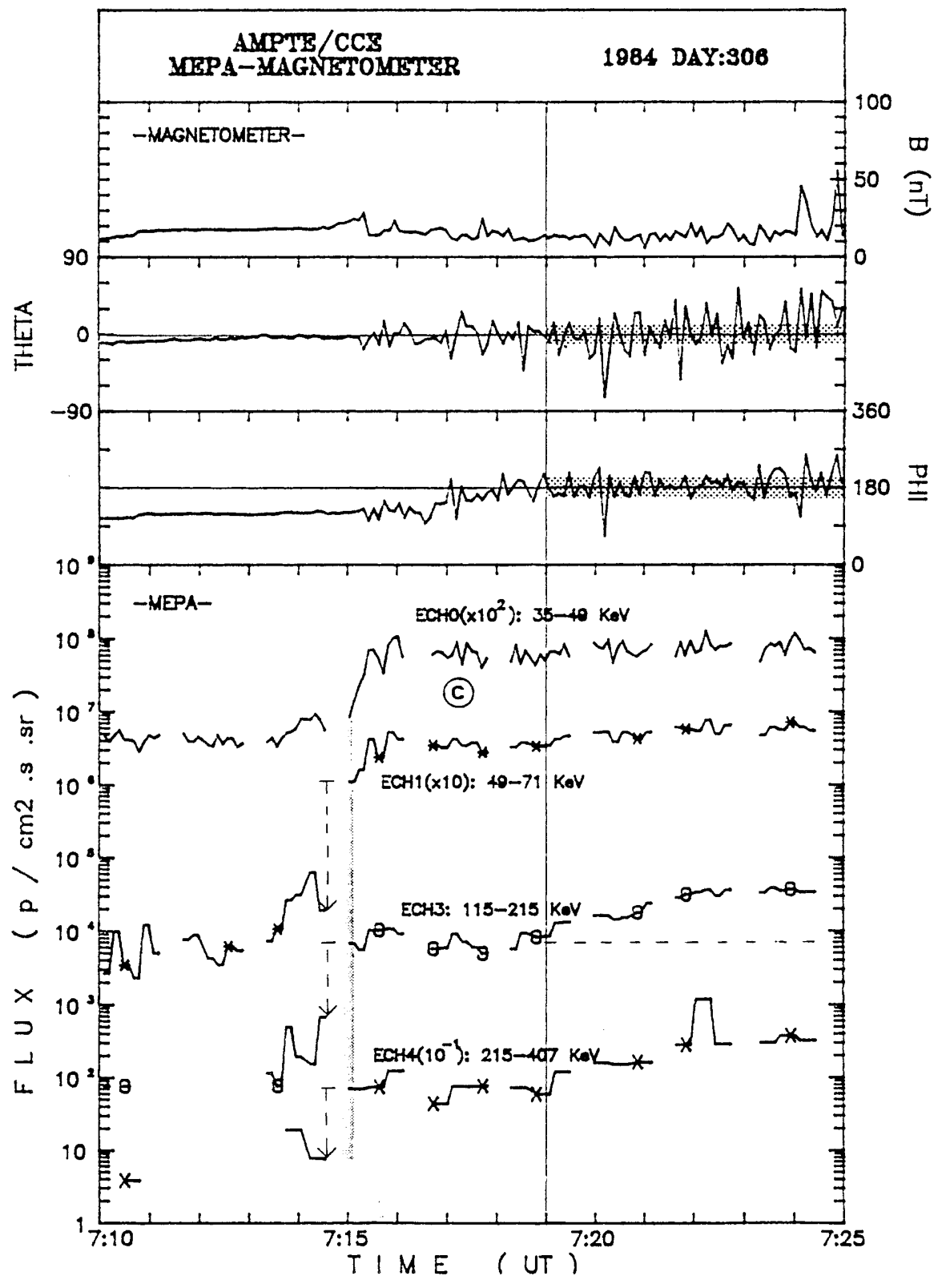

Fig. 3. As in Fig. 2, but for the upstream event (c). Notice that the onset of the middle energies was detected a little earlier than the onset in the lowest energy channel. The normal line $B 1$ indicates the time of onset of the substorm injection event $B$ and suggests the magnetospheric origin of the flux increase at that time
(Krimigis et al., 1985). Furthermore, after $\sim 0720$ UT the following variations have been confirmed: (a) a distinct injection event at the nightside geostationary orbit (Baker et al., 1988; Plate 1, Fig. 3 and 4, and their description in the text), (b) a relaxation of the dayside magnetopause compression (Baker et al., 1988; their Fig. 4), (c) flux and spectral variation of energetic ions in the upstream region (Figs. 3 and 5) and (d) a relativistic $(\mathrm{E} \geq 220 \mathrm{keV})$ electron burst upstream from the bow shock, at the position of IMP-8 (Sibeck et al., 1988). These observations suggest that the upstream spectral variations seen by CCE at $\sim 0720$ UT are well related to a global change of the Earth's magnetosphere at that time, and further confirm that the observations are consistent with a magnetospheric origin for the upstream ions. Moreover, from the data at $\sim 0720$ UT we conclude that the spacecraft detected a sudden spectral variation of the energetic ion population almost simultaneously all over the magnetosphere and the upstream region (Baker et al., 1988) and that, therefore, a comparison between the 0700-0710 UT and the 07170725 UT ion spectra most probably fails.

\subsection{The ion event on November 9, 1975}

In Fig. 6 we show magnetic field and energetic ion data obtained by IMP-8 between 1700-2100 UT on November 9, 1975; the magnetic field data were collected from the Goddard Space Flight Center magnetometer and the energetic ion data are from the EPE/NOAA and the CPME/APL instruments. In the same figure we also show the position of the spacecraft (inset in the top panel). Figure 7 shows the auroral electrojet indices for the whole day November 9, 1975. From Figs. 6 and 7 we conclude that IMP-8 crossed the Earth's bow shock at 


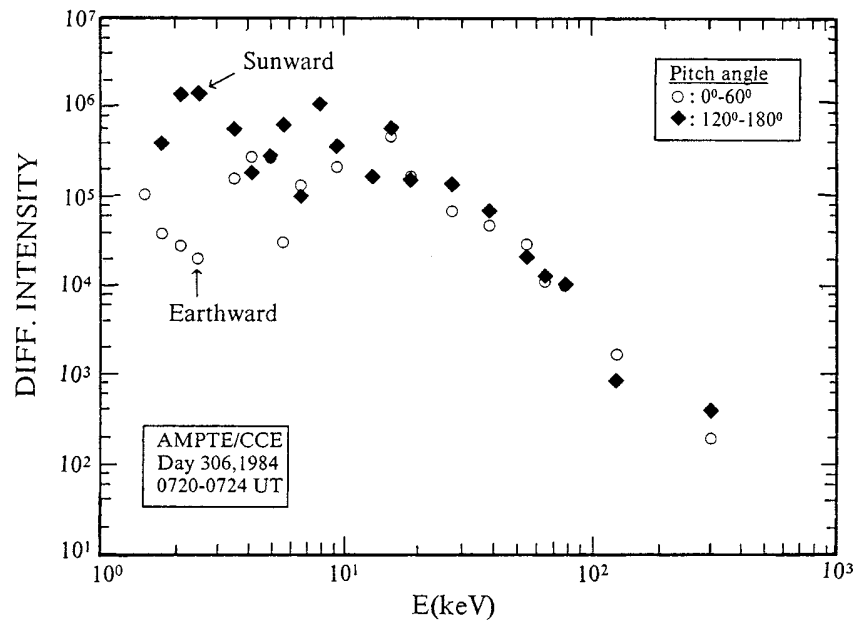

Fig. 4. Differential intensity as a function of energy for protons streaming in the Earthward (open circles) and the Sunward (diamonds) direction during a time period of a radial interplanetary magnetic field connecting with a quasi-parallel bow shock front. The pitch angle distributions suggest the simultaneous detection of two types of proton population: (1) a field aligned beam at low $(\leq \sim 10 \mathrm{keV})$ energies, and (2) a population with broad angular distributions at higher $(\geq \sim 10 \mathrm{keV})$ energies

$\sim 1800$ UT while a series of intense substorms were in progress.

Figure 6 shows that the spacecraft left the magnetosheath, crossed the bow shock and entered the inter- planetary space while $(\sim 1700-\sim 1820$ UT) the direction of the magnetic field changed from a true southward direction $\left(\theta \cong-60^{\circ}\right)$ toward the solar ecliptic plane $\left(\theta \cong 0^{\circ}\right)$. During that time interval the magnetosheath field lines approached the magnetopause. As long as the field lines turned toward the $\mathrm{X}_{\mathrm{SE}}=0$ plane, ions of energies $290-500 \mathrm{keV}$ appeared at the position of IMP-8 first, at $\sim 1730 \mathrm{UT}$, and $50-220 \mathrm{keV}$ ions much later, after a period of $\sim 40 \mathrm{~min}$. The counting rates of the $290-500 \mathrm{keV}$ remained for $\sim 2 \mathrm{~h}$ at an almost constant level, whereas the low energy ion counting rates were strongly dependent on the elevation angle $\theta$ of the IMF (notice the profile of the shaded areas). The dependence of the low energy (50-220 keV) ion intensity on the IMF elevation angle $\theta$ is examined in detail in the next two figures.

Figure 8 displays high time resolution $(\sim 20 \mathrm{~s})$ data of the 50-220 keV counting rate (solid line) and of the IMF elevation angle $\theta$ (solid circles) for the 2-h time interval between 1800-20 UT. From Fig. 8 we see that the 50$220 \mathrm{keV}$ ion intensities become in general highest (i.e., $\mathrm{R} \geq 10$ counts/s) when the angle $\theta$ varies in the range $\sim-8^{\circ} \leq \theta \leq \sim 17^{\circ}$ (the range between the horizontal dashed lines in Fig. 8). For instance, the most intense 50-220 keV ion bursts were observed during the time intervals $\mathrm{B}, \mathrm{D}, \mathrm{F}, \mathrm{H}$ and $\mathrm{L}$ (indicated by normal dashed lines in Fig. 8), while the IMF elevation angle ranged between $\sim 8^{\circ}$ and $\sim 17^{\circ}$. In contrast, the $50-220 \mathrm{keV}$ ions show, in general, low intensities (or even remain at the
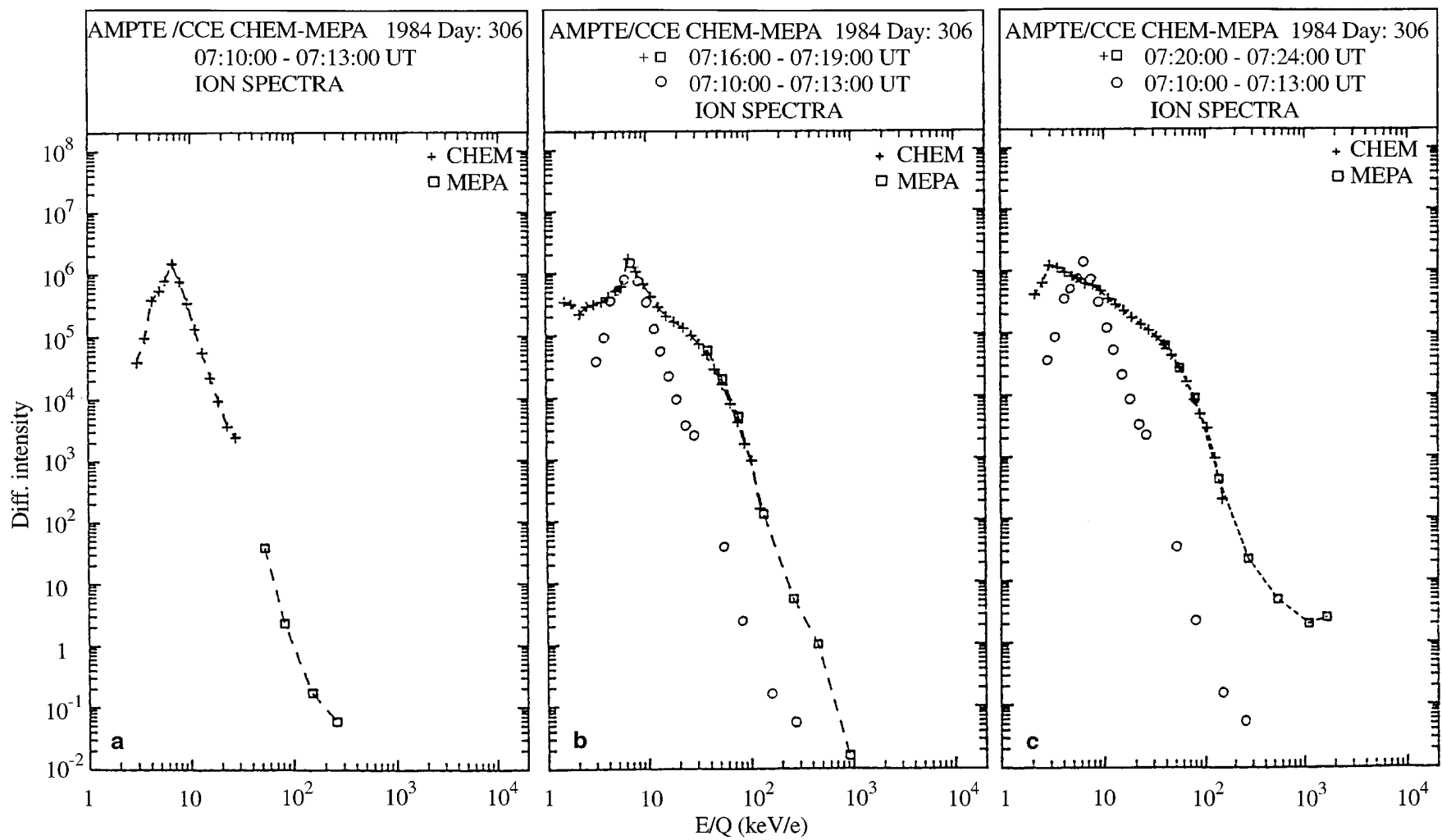

Fig. 5a-c. Energy spectra for three different time periods between 0710-0724 UT on November 1, 1984. A field aligned beam of low energy ions is seen either alone, upstream from the quasi-perpendic-

ular bow shock a or along with a broad angular distribution ion population of higher energies, upstream from the quasi-parallel bow shock b-c 


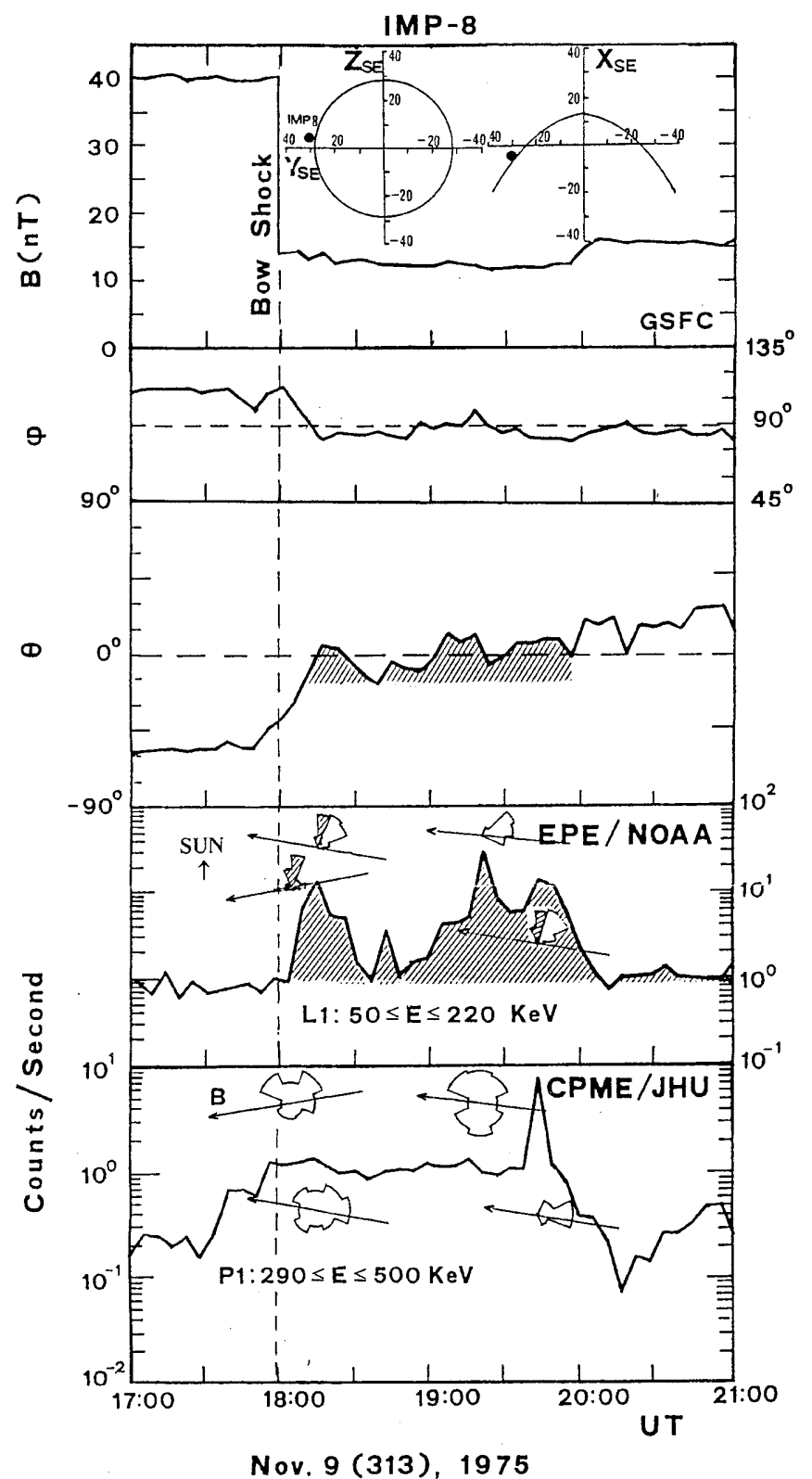

Fig. 6. Magnetic field and energetic ion data, the position of IMP-8 and characteristic angular distributions for the time interval indicated in Fig. 6. The flux of low energy ions are strongly correlated with the direction of the IMF throughout the event. The high energy magnetospheric ions arrived at the position of IMP-8 first, and the low energy ions later (forward velocity dispersion), after a major turning of the IMF

background level of $\sim 2$ count/s) for elevation angle values $\theta \leq \sim-8^{\circ}$ (time intervals $\mathrm{A}, \mathrm{C}, \mathrm{E}$ and $\mathrm{G}$ ) or $\theta>\sim 17^{\circ}$ (time intervals $\mathrm{C}, \mathrm{I}, \mathrm{K}$ and $\mathrm{M}$ ). Figure 8 strongly suggests that the low energy $(50-220 \mathrm{keV})$ ion counting rate $R$ reaches its highest value at the lowlatitude magnetosheath and decreases with increasing (absolute) latitudes, at times of southward or northward turning of the IMF.

Figure 9 displays a scatter plot of the $50-220 \mathrm{keV}$ ion rate versus the magnetic latitude between 1800-2000 UT on November 9, 1975, except for the short time intervals

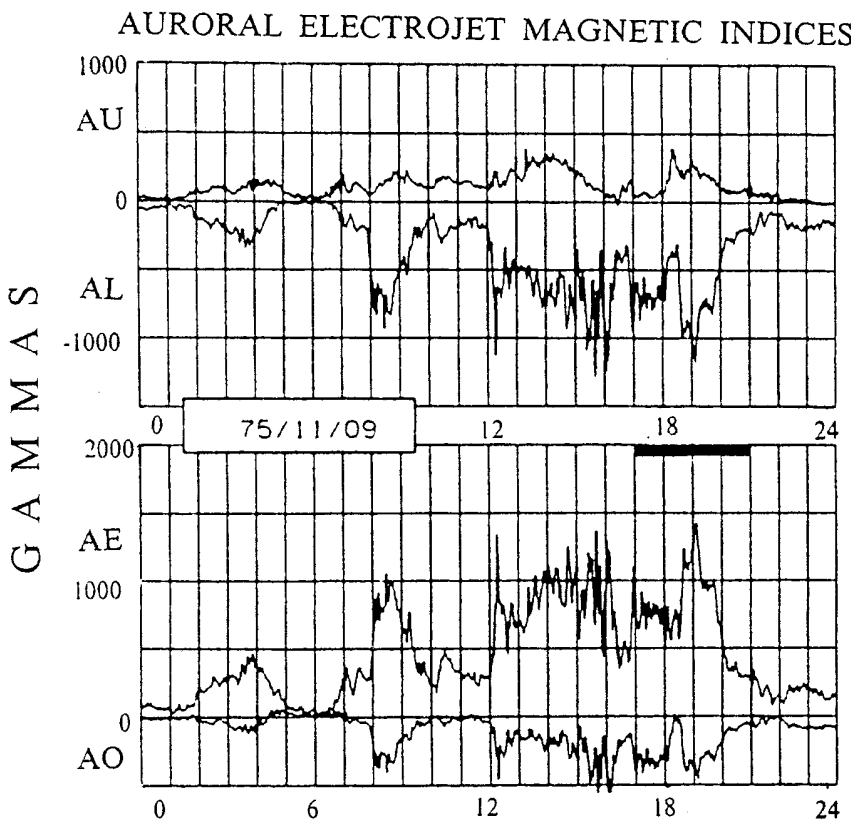

Fig. 7. The auroral electrojet indeces for the day 313, 1975. The solid bar toward the end of the magnetic storm indicates a time interval during which forward velocity dispersion of leaking magnetospheric ions was detected by IMP-8 (Fig. 6)

$\mathrm{C}, \mathrm{I}, \mathrm{K}$ and $\mathrm{M}$ when the IMF turned strongly northward. We see that, for latitudes $\theta \geq 80^{\circ}$, the counting rate $R$ of the low-energy ions decreases as the angle $\theta$ increases. The Speerman correlation coefficient was found to be $r=-0.7$. This indicates that there is a very strong anticorrelation between $R$ and $\theta$ for the time intervals examined, with a critical level of $P<0.001$. The mathematical equation that corresponds to the line in Fig. 8, which was obtained by the method of least squares is given by $R=11955 \exp (-0.077 \theta)$, and suggests that an exponential equation describes well enough the flux variation as a function of magnetic field latitude $\theta$ at high $\theta$ values, whereas it underestimates the flux in the range $\sim 80^{\circ} \leq \theta \leq \sim 90^{\circ}$.

(We confirmed a latitude-dependent $50-220 \mathrm{keV}$ ion flux pattern in the upstream region in almost all the 10 intense upstream magnetospheric ion events we examined in detail, where significant southward or northward turning of the IMF could be seen. In particular, we evaluated the relative intensity gradient

$G_{R}=j^{-1}(\partial j / \partial z)=\left(j_{P}-j_{P}^{\prime}\right) /\left(j_{0} \cdot 2 R\right)$

of 50-220 keV ions from the anisotropy of the intensities $j_{P}$ and $j_{P}^{\prime}$ measured perpendicular to the direction of the IMF ( $j_{0}$ is the mean intensity and $R$ the gyroradius) and we found: (1) the existence of significant intensity gradients toward the plane $\mathrm{Z}_{\mathrm{SE}}=0 R_{\mathrm{E}}$, (2) a change in the direction of the intensity gradient during changes of the IMF from a southward (northward) to a northward (southward) direction, (3) normal values of the relative intensity gradient of $50-220 \mathrm{keV}$ ions $G_{R} \leq \sim 20-30 \%$ / $R_{\mathrm{E}}$, and in some exceptional cases as high as $G_{R}=60$ $100 \% / R_{\mathrm{E}}$, and (4) a trend for detecting a higher intensity gradient when the elevation angle $\theta$ increases. These 


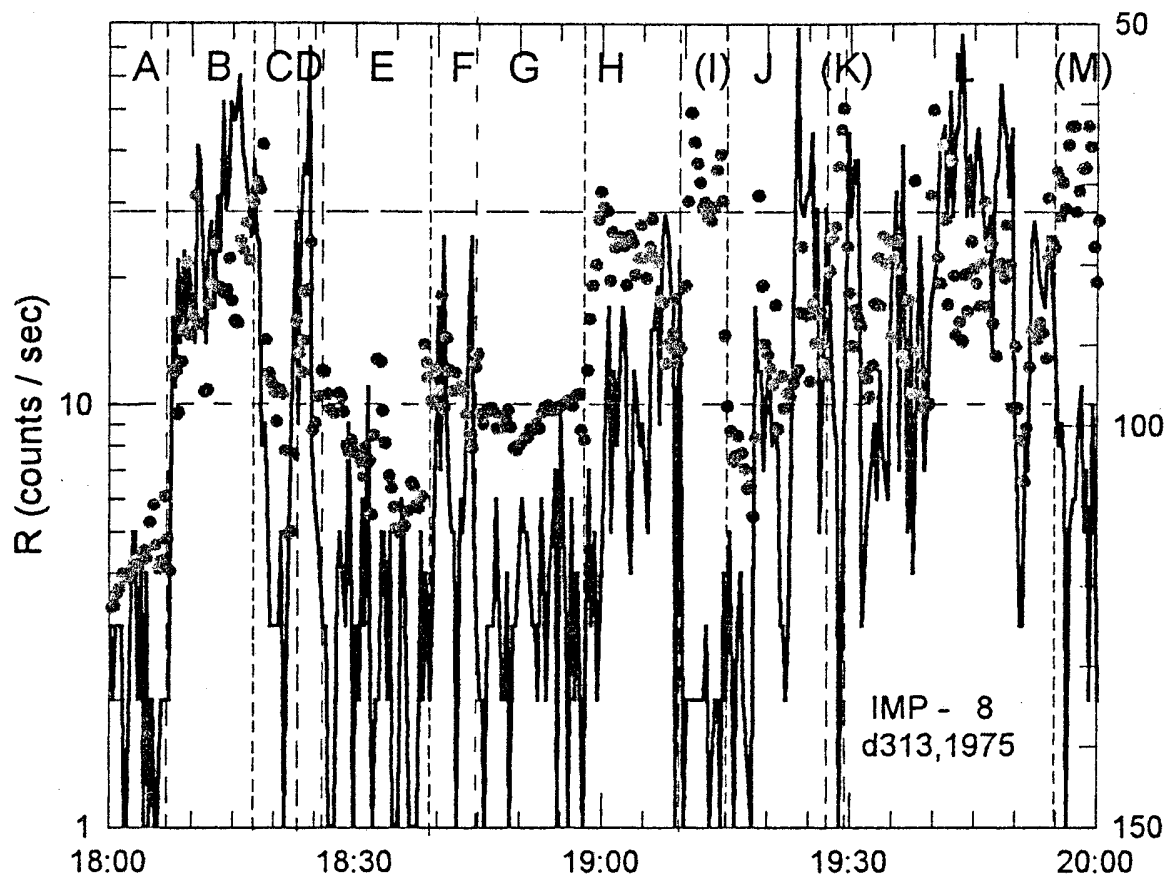

Fig. 8. The time history of $50-220 \mathrm{keV}$ ion counting rate (black line) and of the magnetic field latitude (solid circles) during a time period when the higher energy (290$500 \mathrm{keV}$ ) ion counting rate were constant (Fig. 6). The data suggest that the low energy flux of magnetospheric ions is strongly controlled by magnetic field latitude variations and that it becomes highest near the ecliptic plane, for instance between $\sim-8^{\circ}\left(98^{\circ}\right) \leq \theta \leq \sim 17^{\circ}\left(73^{\circ}\right)$ (see the horizontal dashed lines in the figure). The observations are consistent with preferential leakage of magnetospheric ions from the low latitude magnetopause/bow shock results are consistent with previous relative results of Wibberenz et al. (1985) and Anagnostopoulos et al. (1987), who reported the existence of intensity gradients of $\sim 50 \mathrm{keV}$ ions toward the $\mathrm{Z}_{\mathrm{SE}}=0$ plane with $G_{R}=12.1 \% / R_{\mathrm{E}}$ and $G_{R}=15 \% / R_{\mathrm{E}}$, respectively).

The observations on November 9, 1975 contradict the predictions of bow shock acceleration. Diffusive

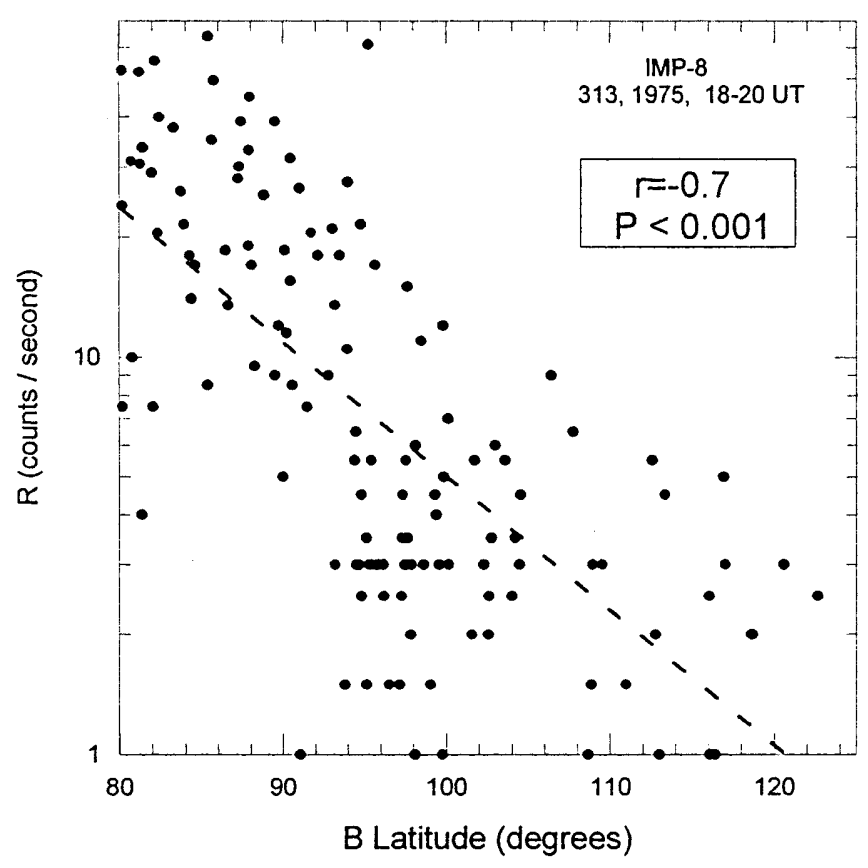

Fig. 9. The counting rate of $50-220 \mathrm{keV}$ ion counting rate as a function of the magnetic field latitude for the data with $\theta>80^{\circ}$ shown in Fig. 8. There is a strong negative relation of the counting rate $R$ with the magnetic field latitude $\theta$; an exponential equation well describes the relation between $R$ and $\theta$, in particular at higher latitudes acceleration, for instance, cannot explain the appearance of the forward velocity dispersion effect at the onset, or the appearance of high energy $(\geq 290 \mathrm{keV})$ ions under extremely short connection times $\left(\varphi_{\mathrm{IMF}} \cong 90^{\circ}\right.$ $\left.-110^{\circ}\right)$. Furthermore, the shock drift acceleration cannot explain the following characteristics: (a) the flux-time profile of the $290-500 \mathrm{keV}$ ions during the main phase of the event (1800-2000 UT), which is independent of the angle $\theta_{B N}$, (b) the detection of higher 50 $220 \mathrm{keV}$ ion fluxes upstream from a quasi-parallel bow shock structure $(\sim 1815 \mathrm{UT})$ rather than upstream from a quasi-perpendicular bow shock structure $(\sim 1800 \mathrm{UT})$, and (c) a high peak-to-background flux ratio $(\mathrm{p} / \mathrm{b} \cong 10)$ of the 290-500 keV ions (see Discussion).

Since energetic ions of energies as high as $\geq 290 \mathrm{keV}$ were observed during a time period of intense geomagnetic activity, we accept that these ions were of magnetospheric origin (Sarris et al., 1978; Anagnostopoulos et al., 1986). Furthermore, the whole set of observations during that time period is consistent with the existence of energy dependent intensity gradients from the bow shock toward the magnetopause, with higher intensity gradients at lower energies. Because of the existence of such gradients, IMP-8 was able to detect increasing counting rates at low energies as long as the IMF lines approached the magnetopause, but almost constant rates at high energies.

Representative angular distributions of $50-220 \mathrm{keV}$ and $290-500 \mathrm{keV}$ ion intensities are seen in the two bottom panels of Fig. 6 . We see that the high energy ions show generally broad distributions, except for the burst over the end of the event (1942 UT), when ions show strong anisotropies along the IMF. The broad distributions of high energy ions are consistent with strong scattering of these ions within the magnetosheath, while the strong anisotropy at the time of the ion burst at 1942 UT is consistent with similar observations 
during the November 1, 1984 events. Furthermore, the Sun-Earth anisotropy of the $290-500 \mathrm{keV}$ ion distributions at $\sim 1805$ is most probably due to the existence of a south-north intensity gradient of the upstream ion population perpendicular to the IMF direction at the position of IMP-8; this scenario is greatly supported by the increase of the ion intensities during the IMF turning toward the $\mathrm{X}_{\mathrm{SE}}=0$ plane (Fig. 6 and earlier relative discussion). The $50-220 \mathrm{keV}$ ion distributions show: (a) strong anisotropies perpendicular to the IMF from the sunward direction, due to the ComptonGetting effect (shaded areas on the distributions) and (b) highest field aligned anisotropy at the time of maximum counting rate $(\sim 1920 \mathrm{UT})$.

Figure 10 displays the energy spectra detected at $\sim 1726$ UT and $\sim 1942$ UT (5.5 min average). The first spectrum (1) is representative of a magnetospheric population leaking almost continuously from certain sites of the bow shock during active magnetospheric periods. The second spectrum (2) in Fig. 10 corresponds to the intense field aligned ion beam at $\sim 1942$ UT and shows an almost power law shape from $\sim 50 \mathrm{keV}$ up to $\sim 3 \mathrm{MeV}$. The latter spectrum is characteristic of a freshly accelerated ion population within the magnetosphere.

\section{Summary of observations}

The analysis of energetic ion data obtained by $\mathrm{CCE} /$ AMPTE and IMP-8 reveal some very interesting

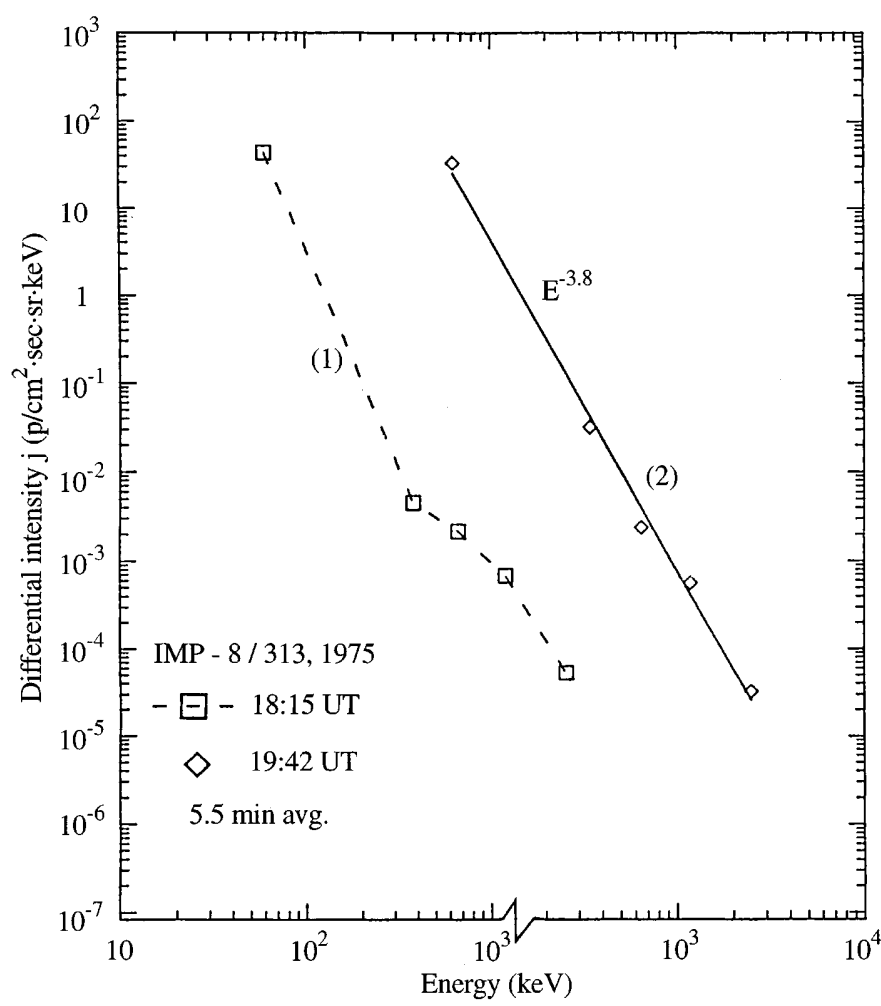

Fig. 10. Energy spectra during the plateau phase (1) and a burst (2) of the $290-500 \mathrm{keV}$ ions characteristics of magnetospheric energetic ions near the bow shock:

\subsection{Flux-time profiles}

a. The flux-time profile of the higher energy ions was found to last longer than the profile of the lower ions; i.e., the higher energy ion flux onset (decay) was observed earlier (later) than the onset (decay) of the lower energy ion fluxes, displaying forward velocity dispersion (Figs. 1, 3 and 6).

b. The time delay between the onset (decay) of the lower and the higher energy ions depends on the time scale of the variation of the IMF direction and on the energies compared. It was found to be as short as $\sim 20$ $40 \mathrm{~s}$ between ions in the energy ranges $35-49 \mathrm{keV}$ and 49-71 keV and as large as $\sim 40 \mathrm{~min}$ between ions in the energy ranges $50-220 \mathrm{keV}$ and $290-500 \mathrm{keV}$ respectively (Figs. 1, 3 and 6).

c. The flux-time profiles of the upstream energetic $(\geq \sim 35 \mathrm{keV})$ ions are strongly related to variations of the IMF direction and the substorm ion injection activity at the nightside geostationary orbit.

d. The energetic $(\geq 35 \mathrm{keV})$ ions present before the generation of magnetic field waves in the upstream region (Figs. 1 and 3).

\subsection{Energy spectra}

a. The ion spectra display a characteristic hump at middle (49-115 keV) energies, at both the onset and the decay phase of upstream events, with a ratio of the event-to-pre-event ion fluxes as high as $R \geq 400$.

b. The energy spectra during the main (plateau) phase of the event show a clear power law shape in the energy range 50-70 $\leq \mathrm{E} \leq 2000-3000 \mathrm{keV}$.

c. The energy spectra display a variety of forms between the two kinds of spectra just described (2a and b) and often resemble the spectral forms reported earlier by Ipavich et al. (1981) and Anderson et al. (1981).

d. A peak at $\sim 6 \mathrm{keV}$ is a common characteristic of the upstream spectrum in a broad range of angle $\theta_{\mathrm{Bn}}$ (from almost perpendicular to almost parallel).

\subsection{Angular distribution of ion fluxes}

a. The angular distributions of upstream energetic $(\geq 50 \mathrm{keV})$ ions were anisotropic during the onset phase and at times of intensity enhancements throughout the event; they displayed broad forms at other times.

b. The low energy ion $(\mathrm{E}<\sim 6 \mathrm{keV})$ population preserved a beam-like configuration with a peak-tobackground ratio as large as $10^{2}$ upstream from both a quasi-perpendicular and a quasi-parallel bow shock as long as the higher energy ions $(E \geq 6 \mathrm{keV})$ showed broad angular distributions. 


\section{Discussion}

In this study we analyzed high time resolution ion data, in a broad energy range, from $\sim 0.01$ to $\sim 2.00 \mathrm{MeV}$, in order to elaborate the form of the flux-time profile, the energy (velocity) dispersion and the energy spectrum of ion events near the Earth's bow shock, in particular at the onset and the decay phase. The analysis of high time resolution data reveals that the flux-time profile of ion events is often longer at lower energies than at higher energies.

\subsection{Longer duration profiles of higher energy ions}

The most striking finding of this study is the longer intensity-time profile of the higher energy ions. In all of the three successive events observed by CCE on November 1, 1984, we found FVD at their onset phase, i.e., appearance of the higher $(\geq 49 \mathrm{keV})$ energy ions before the appearance of the lower (35-49 keV) energy ions; in one of these events (event a) the high energy flux-time profiles remained at high levels even after the fall of the low energy ions at the pre-event flux level (for the two other events there was some data gap at the decay phase).

There are several processes which could explain an energy dispersion of ion intensities near the bow shock (Anagnostopoulos et al., 1994):

a. An $\mathbf{E} \times \mathbf{B}$ drifting of various velocity ions escaping from the bow shock. This process could explain the FVD at the onset of events (Anderson, 1981), but it cannot account for the FVD of the ion events observed downstream from the bow shock.

b. During times of varying IMF, magnetospheric ions reaching sites of the bow shock of various angle $\theta_{\mathrm{B}_{\mathrm{n}}}$ (the angle between the IMF direction $\mathbf{B}$ and the normal to the shock front $\mathbf{n}$ ), escape with different energies and produce FVD. This scenario cannot explain the downstream observations as well.

c. FVD could be produced both in the upstream region and the magnetosheath, as a result of the time delay between particles of different velocities (Taktakishvili et al., 1989). This process cannot explain the observations during the decay phase of the events.

d. A discontinuity in ion intensity $\mathbf{j}$ at an almost perpendicular region of the bow shock, due to the presence of a magnetospheric population in the magnetosheath, can produce a grad $\mathbf{j} \times \mathbf{B}$ anisotropy just upstream from the bow shock and longer profiles in higher energy ion flux-time profiles. This scenario cannot explain the energy dependent duration of the ion profiles in the magnetosheath as well as the long time delays between the onset (decay) times of various energy ions.

e. A rigidity dependent transport process within the magnetosheath (see later). Such a model can account for the new observations.

Pavlos et al. (1985) confirmed a rigidity dependent process for the leakage of the $\geq 50 \mathrm{keV}$ ions from their source, the plasma sheet, to the magnetosheath. In addition, Sarris et al. (1978) found good evidence that energetic ions propagate via a rigidity dependent leakage process in the whole area from within the plasma sheet up to interplanetary space. Finally, in our Fig. 8 we schematically suggest a scenario which may explain the production of a longer flux-time profile of higher energy ions near the bow shock based on the assumption of a rigidity dependent leakage process for energetic particles in the magnetosheath.

Magnetospheric ions, after their escape from the magnetopause, scatter and propagate within the magnetosheath. Ions of higher energy and larger gyroradius, are distributed over a more extensive area than the low energy ions; consequently, they produce smoother intensity gradients than the low energy ions. If at the position of a detecting spacecraft an IMF of a variable direction arrives (Fig. 11b), ions will escape from various sites of the magnetosheath in the upstream region and will produce different flux-time profiles at various energies (Fig. 11a). More explicitly, when the IMF turns and a field line contacts the quasi-perpen-
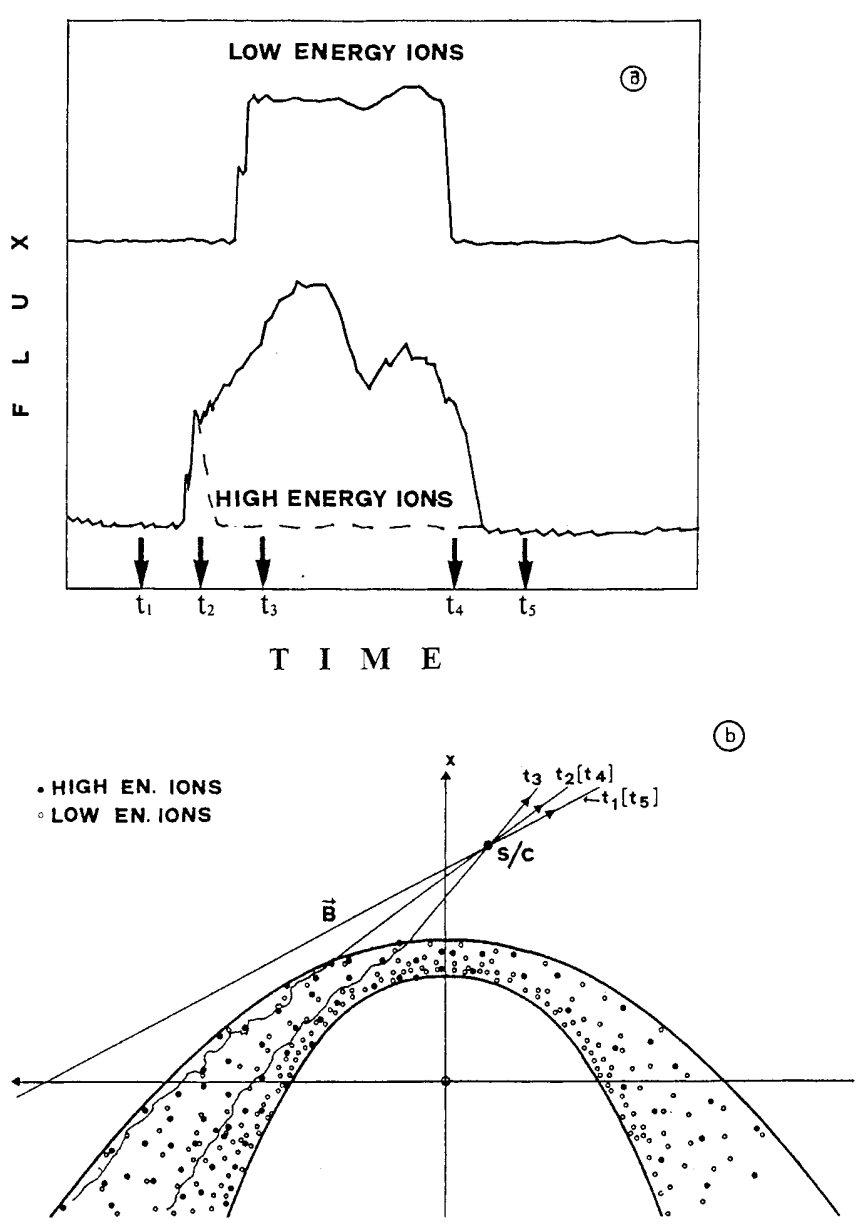

Fig. 11. Schematic indicating how forward velocity dispersion can be detected in the upstream region as a result of energy dependent intensity gradients of magnetospheric protons within the magnetosheath. The higher energy protons are distributed over a large area and are observed first, when the detecting spacecraft is connected magnetically with the bow shock 
dicular bow shock (Fig. 11; direction: 2, time: $\mathrm{t}_{2}$ ), the higher energy ions, which are presumably distributed all over the magnetosheath, will follow the field line and will reach the spacecraft; then a high energy ion flux onset will be observed. If the IMF turns back towards its first direction (1), the ion intensities fall and a burst only at high energies will be observed by the detecting spacecraft (dashed line in Fig. 11a). Alternatively, if the IMF direction continues to turn and connects the upstream spacecraft with points in the magnetosheath closer to the magnetopause (Fig. 11, direction: 3, time: $t_{3}$ ), where a significant amount of low-energy ions exist, a part of the low-energy ion population will escape and will arrive at the position of the spacecraft causing the onset of the low-energy ion fluxes. If, at later times, the IMF turns toward its first direction (or further turns towards dawn), the field lines will move increasingly further away from the magnetopause (Fig. 11; direction: 2, time: $t_{4}$ ), and the low-energy ion fluxes will fall in the magnetosheath and, consequently, in the upstream region. At even later times (Fig. 11; direction: 1, time: $t_{5}$ ) the high-energy ion fluxes will fall as well, showing a delay with respect to the decay time of the low-energy ion fluxes. This scenario explains the longer profiles of higher energy ions as a spatial effect, although other processes producing FVD (see earlier) may operate at the same time.

We would like to argue here that the arrival of the high-energy $(\geq 50 \mathrm{keV})$ ions that was observed earlier than the onset of the low-energy ions in the upstream region on November 1, 1984 cannot be attributed to shock drift acceleration (SDA). As far as we know the strongest acceleration effects interpreted in terms of SDA at the Earth's bow shock have been discussed in some of our previous studies (Anagnostopoulos and Kaliabetsos, 1994 and references therein). In these studies we demonstrated that the peak-to background flux ratio of $50-220 \mathrm{keV}$ ions detected by IMP-8 near quasi-perpendicular regions of the bow shock was $\sim 3$ under the most favorable conditions. This value could be a little higher for an ion channel in the energy range $\sim 50-70 \mathrm{keV}$ (instead of $50-220 \mathrm{keV}$ ) under the same favorable conditions. However, during the interval examined here on November 1, 1984, there was no preexisting energetic ion population of solar origin; for instance, the background ion flux of solar origin was $j \cong 10^{-3} \mathrm{p} / \mathrm{cm}^{2} \cdot \mathrm{s} \cdot \mathrm{sr} \cdot \mathrm{keV}$ for $\sim 300 \mathrm{keV}$ ions instead of $\sim 20 \mathrm{p} / \mathrm{cm}^{2} \cdot \mathrm{s} \cdot \mathrm{sr} \cdot \mathrm{keV}$ in the study of Anagnostopoulos and Kaliabetsos (1994).

Figure $12 \mathrm{a}$ shows the results from a calculation of the final-to-ambient proton intensity ratio $J_{F} / J_{A}$ predicted for a single encounter SDA at a planar MHD shock as a function of the angle $\theta_{B N}$. The calculation was based on the analysis of Decker (1983) and it was derived for values of the solar wind speed $\mathrm{V}=450 \mathrm{~km} / \mathrm{s}$, angle between the direction of the solar wind and the normal at the shock front $0^{\circ}$ and jump ratio of magnetic field $N=2.5$. It is evident that the predicted values of the ratio $J_{F} / J_{A}$ vary as a function of the characteristic angle $\theta_{B N}$ and are dependent on the spectral index $\gamma$ (for a power law spectral form: $\left.j \sim \mathrm{E}^{-\gamma}\right)$. For values of the
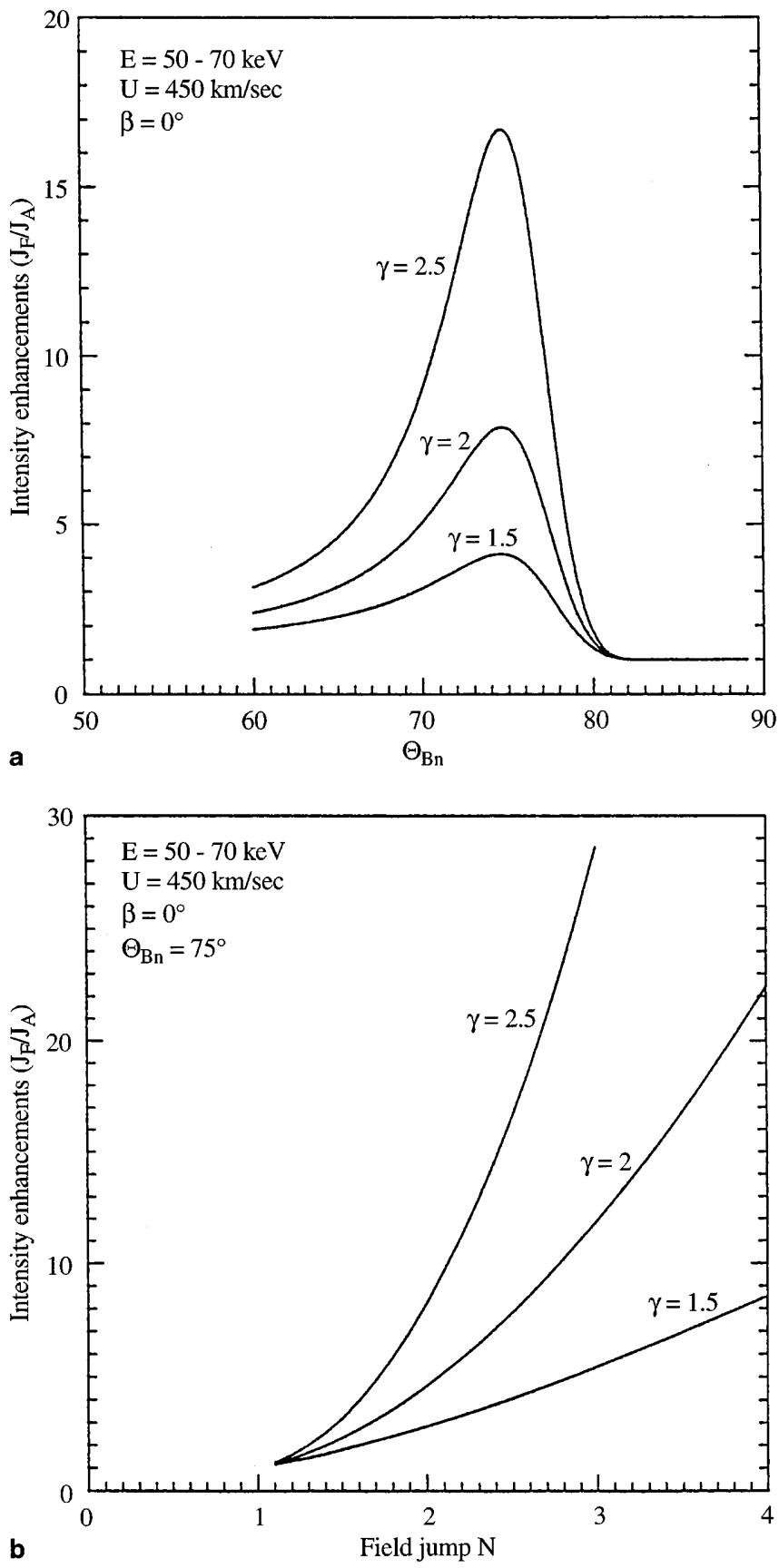

Fig. 12a, b. The predicted intensity enhancements for a power law proton distribution of energy $50-70 \mathrm{keV}$ accelerated via shock drift acceleration (Decker, 1983) as a function of $\mathbf{a} \theta_{B N}$ angle and $\mathbf{b}$ the ratio $N$ of downstream-upstream magnetic field, for three values of the spectral index Y. The predicted values are much less than the observed ones (Figs. 3, 6 and 7)

spectral index $\gamma=1.5,2$ and 2.5 used in our calculation, we see that the predicted maximum ratio $J_{F} / J_{A}$ is $\sim 18$. In addition, Fig. $12 \mathrm{~b}$ shows that the ratio $J_{F} / J_{A}$ does not increase significantly as a function of the jump ratio $N$. Thus, the calculations presented in Fig. 12 confirm that SDA can account for $\sim 50 \mathrm{keV}$ proton intensity enhancements $(\mathrm{p} / \mathrm{b} \cong 3)$ observed by IMP-8 (Anagnostopoulos and Kaliabetsos, 1994). However, the intensity enhancement $J_{F} / J_{A}$ of the $\sim 50-70 \mathrm{keV}$ ions 
observed at CCE ranged between 250-1250, which is much higher than the predicted values. Furthermore, there was no appropriate ambient proton population of solar origin, which could explain values of the ratio $J_{F} / J_{A}=20-30$.

\subsection{Forward or inverse velocity dispersion?}

Although a large number of upstream ion events have been published in a series of papers for more than 20 years, only recently has FVD been emphasized as a usual characteristic of energetic ions in the vicinity of Earth's and Jupiter's bow shock (Anagnostopoulos et al., 1995). Our examination of a large array of ion events near the bow shock reveals that FVD can be seen well only if: (a) high time resolution data are used, (b) fluxes from ion channels of comparative energies are examined (ions of very different energies observed by detectors of different noise thresholds can obscure the initial forward velocity dispersion), and (c) the onsets of various energy ion intensities are compared, instead of the times of achievement of the plateau phase.

\section{Conclusions}

We have mentioned that the high time resolution data used in this study reveals new features of the upstream energetic ion population. Based on the new ion characteristics we have suggested a rigidity dependent fast cross-field charged particle transport process within the magnetosheath. This process can produce two types of ion events near the Earth's bow shock: (a) bursts of only high energy ions, and (b) ion events with longer ion fluxtime profiles at higher energies than at lower energies. It can also explain the generation of some high-energy ion bursts in the vicinity of Jupiter's bow shock reported by Zwickl et al. (1981).

The present study confirms that various kinds of $\geq 30 \mathrm{keV}$ ion angular distributions (FABs and BADs) and of ion spectra (from a shape with a hump at energies $50 \leq \mathrm{E} \leq 215 \mathrm{keV}$ up to one of a power law between $\sim 50-\sim 2000 \mathrm{keV}$ and other intermediate shapes) can be explained as reflecting different stages in the leakage process of magnetospheric ions. It is interesting to note that some of the ion spectra of the events analyzed resemble the ion spectra reported by Ipavich et al. (1981b) and Anderson (1981).

The whole data set analyzed in this study reveals a new picture for magnetospheric ion events observed upstream and downstream from the Earth's bow shock. The new picture became possible due to the high time resolution data $(6 \mathrm{~s})$ used for studying the onset and the decay of upstream energetic ( $\geq 30 \mathrm{keV})$ ion events and, we believe, opens up a new perspective in the study of energetic ion events in the vicinity of the Earth's bow shock. It is of great importance for future studies to define the extent to which this new picture of ion events is valid.

Acknowledgements. We would like to thank Drs R.W. McEntire and $\mathrm{G}$. Gloeckler for making available high time resolution ion data from their experiments on board the spacecraft $\mathrm{CCE} /$ AMPTE. We also thank Drs S.M. Krimigis, D.C. Williams and R.P. Lepping for making available the energetic particle (CPME/ APL-EPE/NOAA) and the magnetic field (GSFC) data from IMP-8. In the data processing we have been supported by Dr I. Daglis, G. Kaliabetsos and S. Raptis and we acknowledge their help; we are also grateful for helpful discussions with Prof. E.T. Sarris. This work has been partially supported by the contract PENED 95-4.21783 of the Secretariat for Science and Technology of the Greek Ministry for Development.

Topical Editor K.-H. Glassmeier thanks two referees for their help in evaluating this paper.

\section{References}

Anagnostopoulos G. C., Dominant acceleration processes of energetic protons at the Earth's bow shock, Phys. Scri., T52, 142$151,1994$.

Anagnostopoulos, G. C., and G. D. Kaliabetsos, Shock drift acceleration of energetic (E»50 keV) protons at the Earth's bow shock as a source of the magnetosheath energetic proton events, J. Geophys. Res., 99, 2335-2349, 1994.

Anagnostopoulos, G. C., E. T. Sarris, and S. M. Krimigis, Magnetospheric origin of energetic $(E \gg 50 \mathrm{keV})$ ions upstream of the bow shock: the October 31, 1977 event, J. Geophys. Res., 91, 3020-3028, 1986.

Anagnostopoulos, G. C., E. T. Sarris, and S. M. Krimigis, On the origin of the forward velocity dispersion of ion events observed near the Earth's and Jupiter's bow shock, Adv. Space Res., 16, (4), (4)49-(4)152, 1995.

Anagnostopoulos, G. C., G. Kaliabetsos, G. Argyropoulos, and E. T. Sarris, Upstream energetic (50-220 keV) ion events associated with high energy electron $(\geq 220 \mathrm{keV})$ and ion $(\geq 290 \mathrm{keV})$ bursts: six years (1982-1986) statistical results, Geophys. Res. Lett., 26, 2151, 1999.

Anderson, K. A., Measurements of bow shock particles far upstream from the Earth, J. Geophys. Res., 86, 4445-4454, 1981.

Baker, J. B., R. D. Belian, T. A. Fritz, P. R. Higbie, S. M. Krimigis, D. G. Sibeck, and R. D. Zwickl, Simultaneous energetic particle observations at geostationary orbit and in the upstream solar wind: evidence for leakage during the magnetospheric compression event of November 1, 1984, J. Geophys. Res., 93, 14315, 1988.

Decker, R. B., Formation of shock-spike events at quasi-perpendicular shocks, J. Geophys. Res., 88, 9959, 1983.

Ellison, D. C., E. Mobius, and G. Paschmann, Particle injection and acceleration at Earth's bow shock: comparison of upstream and downstream events, The Astrophys. J., 352, 376-394, 1990.

Giacalone, J., Shock drift acceleration of energetic protons at a planetary bow shock, J. Geophys. Res., 97, 8307-8318, 1992.

Gosling, J. T., J. R. Asbridge, S. J. Bame, G. Paschmann, and N. Sckopke, Observations of two distinct populations of bow shock ions in the upstream region, Geophys. Res. Lett., 5, 957, 1978.

Ipavich, F. M., M. Scholer, and G. Gloeckler, Temporal development of composition, spectra, and anisotropies during upstream particle events, J. Geophys. Res., 86, 11 153, 1981.

Ipavich, F. M., G. Gloeckler, D. C. Hamilton, L. M. Kisler, and J. T. Gosling, Protons and alpha particles in field-aligned beams upstream of the bow shock, Geophys. Res. Lett., 15, 1153-1156, 1988.

Karanikola, I., G. C. Anagnostopoulos, and A. G. Rigas, Characteristics of $\geq 290 \mathrm{keV}$ magnetosheath ions, Ann. Geophysicae, 17, $5,1999$.

Krimigis, S. M., G. C. Anagnostopoulos, E. T. Sarris, K. Kudela, M. Slivka, S. Fisher, M. Vandas, and N. Paschalidis, Simultaneous observation of ion spectra evolution within the magnetosphere, magnetosheath and upstream region during magnetospheric substorms, EOS, 75, 570, 1995. 
Kudela, K., D. G. Sibeck, R. D. Belian, S. Fischer, and V. Lutsenko, Possible leakage of energetic particles from the magnetosphere into the upstream region on June 7, 1985, J. Geophys. Res., 95, $20825,1992$.

Lee, M. A., Coupled hydromagnetic wave excitation and ion acceleration upstream of the Earth's bow shock, J. Geophys. Res., 87, 5063, 1982.

Mobius, E., Energetic particles in the environment of the Earth's magnetosphere, J. Atmos. Ter., 52, 1169, 1990.

Paschalidis, N. P., E. T. Sarris, S. M. Krimigis, R. W. McEntire, M. D. Levine, I. A. Daglis, and G. C. Anagnostopoulos, Energetic ion distributions on both sides of the Earth's magnetopause, J. Geophys. Res., 99, 8687-8703, 1994.

Pavlos, G. P., E. T. Sarris, and G. Kaliabetsos, Monitoring of energy spectra of particle bursts in the plasma sheet and magnetosheath, Planet. Space Sci., 33, 1109-1118, 1985.

Sarris, E. T., S. M. Krimigis, C. O. Bostrom, and T. P. Armstrong, Simultaneous multispacecraft observations of energetic proton and electron bursts inside and outside the magnetosphere, $J$. Geophys. Res., 83, 4289, 1978.

Scholer, M., D. Hoverstadt, F. M. Ipavich, and G. Gloekler, Upstream energetic ions and electrons: bow shock-associated or magneto-spheric origin?, J. Geophys. Res., 86, 9040, 1981.

Scholer, M., K. J. Trattner, and H. Kucharek, Ion injection and Fermi acceleration at Earth's bow shock: the 1984 September 12 event revisited, The Astrophys. J., 395, 675-681, 1992.

\section{Note added in proof}

Wibberenz, G., F. Zollich, H. M. Fischer, and E. Keppler, Dynamics of intense upstream ion events, J. Geophys. Res., 90, 283, 1985.
Sibeck, D. G., R. W. McEntire, S. M. Krimigis, and D. N. Baker, The magnetosphere as a sufficient source for upsream ions on November 1, 1984, J. Geophys. Res., 99, $14328,1988$.

Tanaka, M., C. C. Goodrich, D. Winske, and K. Papadopoulos, A source of backstreaming ion beams in the foreshock region, $J$. Geophys. Res., 88, 3046, 1983.

Takahashi, S., and T. Iyemori, Three-dimensional tracing of charged particle trajectories in a realistic magnetospheric model, J. Geophys. Res., 94, 5505, 1989.

Takahashi, K., L. M. Kistler, T. A. Potemra, R. W. McEntire, and L. J. Zanetti, Magnetospheric ULF waves observed during the major magnetospheric compression of November 1, 1984, J. Geophys. Res., 93, A12, $14369,1988$.

Taktakishvili, A. L., L. M. Zelenyi, E. T. Sarris, R. E. Lopez, and D. V. Sarafopoulos, Temporal dispersion structures of proton and electron bursts in the Earth's magnetotail, Planet. Space Sci., 41, 461, 1993.

Williams, D. T., D. G. Mitchell, L. A. Frank, and T. E. Eastman, Three-dimensional magnetosheath plasma ion distributions from $200 \mathrm{eV}$ to $2 \mathrm{MeV}$, J. Geophys. Res., 93, 12 783-12 794, 1988.

Zwickl, R. D., S. M. Krimigis, J. F. Carbary, E. P. Keath, T. P. Armstrong, D. C. Hamilton, and G. Gloeckler, Energetic particle events $(\geq 30 \mathrm{keV})$ of Jovian origin observed by Voyager 1 and 2 in interplanetary space, J. Geophys. Res., 86, 8125-8140, 1981. 